\title{
STANDARD ERRORS FOR THE HARRIS-KAISER CASE II ORTHOBLIQUE SOLUTION
}

\author{
Haruhiko Ogasawara*
}

Asymptotic standard errors of the estimates of the obliquely rotated parameters by the Harris-Kaiser Case II orthoblique method are derived under the assumption of the multivariate normal distribution for observed variables. A covariance structure model for observed variables is constructed such that both unrotated and orthogonally rotated parameters are involved in the model. The asymptotic standard errors for the final oblique solution (orthoblique solution) are derived by a stepwise method. First, the asymptotic variance-covariance matrix for the estimates of the unrotated and orthogonally rotated parameters is derived. Second, the delta method is used to obtain the asymptotic variances of the estimates of the obliquely rotated parameters. Results by simulation indicate that the theoretical values of the asymptotic standard errors are close to simulated ones.

\section{Introduction}

In factor analysis, principles of deriving the asymptotic standard errors for the estimates of rotated parameters (factor loadings and correlations) were given by Archer and Jennrich (1973) (see also Jennrich, 1974), and Jennrich (1973) for orthogonal and oblique rotations, respectively. For actual orthogonal rotations, Archer and Jennrich (1973) showed the asymptotic standard errors for the raw orthomax solution and Ogasawara (1996) gave those for the normalized orthomax method. For oblique rotations, Jennrich (1973) provided the asymptotic standard errors of the estimates of rotated parameters by a generalized version of the Crawford-Ferguson family which includes the raw direct-oblimin method as a special case. Ogasawara (1998) and Ogasawara (1999) gave the asymptotic standard errors for the promax solution and various solutions of the procrustes rotation, respectively. Browne and Du Toit (1992) gave the asymptotic standard errors of rotated factor loadings using a numerical derivative. (For actual statistical tests using the standard errors of rotated parameters, see Cudeck \& O'Dell, 1994.)

The purpose of this paper is to derive the asymptotic standard errors for parameter estimates by the Harris-Kaiser Case II orthoblique method (Harris \& Kaiser, 1964) with the assumption of the multivariate normal distribution for observed variables. The Harris-Kaiser orthoblique method is one of the prominent oblique rotation methods for obtaining simple patterns in addition to the direct oblimin (especially direct quartimin) and promax methods (Hakstian, 1971; Hakstian \& Abell, 1974: Harman, 1976, section 14.6). The Harris-Kaiser orthoblique solution has three variants (Cases I. II and III), which will be explained in the

Key Words and Phrases: orthoblique solution, factor rotation, asymptotic standard errors, infomation matrix.

${ }^{*}$ Department of Information and Management Science, Otaru University of Commerce, 35-21, Midori, Otaru 047-8501 Japan. E-mail : hogasa@res.otaru-uc.ac.jp 
second section. Among the orthoblique solutions, only the Case II solution is currently used and is included in the statistical packaged program SAS (SAS Institute Inc., 1990). The Case II orthoblique method is not a special obliquerotation method with particular purposes such as the procrustes rotation method with a target matrix. Note that the Case II orthoblique method is one of the rotation methods for deriving simple patterns. Recently, the orthoblique method (especially the independent cluster solution) has attracted attention in the field of components analysis (Kiers \& Ten Berge, 1994: Kiers. 1997). The method in this paper can be applied to the results of components analysis as well as to those for factor analysis.

The method of deriving the asymptotic standard errors for the parameter estimates of the Case II solution is based on Jennrich (1974). That is, the standard errors are obtained from an augmented information matrix for the parameters with restrictions to identify a factor analysis model. However, since it is difficult to apply this method directly to the orthoblique solution, the rotated solution is reparameterized so that the method of the augmented information matrix can be applied. The asymptotic standard errors of the final solution is obtained by using the delta method (see e.g., Efron \& Tibshirani, 1993, Section 21.9) and the relationships between the final solution and the reparameterized solution.

\section{The Orthoblique Method}

The orthoblique method is based on the following principle. Let $p$ and $k$ $(k<p)$ denote the numbers of observed variables and common factors in an exploratory factor analysis model, respectively; and $\Sigma(p \times p)$ be a covariance matrix for manifest variables. Then, $\Sigma$ is written as:

$$
\Sigma=\Gamma+\Psi
$$

where $\Gamma$ is a nonnegative definite matrix with rank $k$ and $\Psi(p \times p)$ is a diagonal matrix with the diagonal elements being the variances of unique factors. The Case II orthoblique rotation utilizes the following decomposition of $\Gamma$ in (1) followed by stepwise transformations and rescalings:

$$
\begin{aligned}
\Gamma & =Q M^{2} Q^{\prime}=Q M(Q M)^{\prime} \\
& =Q D_{2} T D_{1}\left(D_{1}^{-1} T^{\prime} D_{2}^{-1} M^{2} D_{2}^{-1} T D_{1}^{-1}\right) D_{1} T^{\prime} D_{2} Q^{\prime}
\end{aligned}
$$

(Harris \& Kaiser, 1964: see also Harman, 1976, section 14.6), where $Q(p \times k)$ is a matrix whose columns are the normalized eigenvectors of $\Gamma$ (i.e., $Q^{\prime} Q=I_{k}$ and $I_{k}$ is a $k \times k$ identity matrix): $M^{2}$ is a diagonal matrix with the diagonal elements being equal to the eigenvalues of $\Gamma$ arranged in descending order; $T(k \times k)$ is an ortho-normal matrix (i.e., $T^{\prime} T=I_{k}$ ): and $D_{1}(k \times k)$ and $D_{2}(k \times k)$ are diagonal matrices. The matrix $D_{2}$ is a scaling matrix for the ortho-normal matrix $Q$. This scaling gives a new unrotated matrix $Q D_{2}$, which is further orthogonally 
rotated by the matrix $T$. These stepwise transformations of $Q$ give an oblique solution with the factor covariance matrix $T^{\prime} D_{2}^{-1} M^{2} D_{2}^{-1} T$. The diagonal elements of $D_{1}$ are the standard deviations of the obliquely rotated factors, that is, $D_{1}=\left[\operatorname{Diag}\left(T^{\prime} D_{2}^{-1} M^{2} D_{2}^{-1} T\right)\right]^{1 / 2}$, where $\operatorname{Diag}(\cdot)$ indicates a diagonal matrix whose diagonal elements are those of the parenthesized matrix. Consequently, $B=Q D_{2} T D_{1}$ and $\Phi=D_{1}^{-1} T^{\prime} D_{2}^{-1} M^{2} D_{2}^{-1} T D_{1}^{-1}$ are the loading and correlation matrices of the obliquely rotated factors.

When $D_{2}=M,(2)$ is reduced to

$$
\Gamma=Q M T T^{\prime} M Q^{\prime}
$$

This is the case of ordinary orthogonal factor rotation, where $Q M$ is an unrotated loading matrix and $T$ is a transformation matrix, The model of (3) is called Case I. The matrix $T$ in (2) gives possibly a simple pattern $Q D_{2} T$. In the case of $D_{2}=I_{k}=M^{0}$, the obliquely rotated loading matrix $B$ is $Q T D_{1}$ and $B^{\prime} B$ becomes a diagonal matrix $D_{1}^{2}$. From this property, the case with $D_{2}=I_{k}$ is called the independent cluster solution. When $D_{2}=M^{1 / 2}$, we have $B=Q M^{1 / 2} T D_{1}$, which gives

$$
B^{\prime} B=D_{1} T^{\prime} M T D_{1}
$$

and

$$
\Phi=D_{1}^{-1} T^{\prime} M T D_{1}^{-1}=D_{1}^{-2}\left(B^{\prime} B\right) D_{1}^{-2} .
$$

Since $B^{\prime} B$ is a rescaled matrix of $\Phi$, this is called the proportional solution. In Case II a general solution which includes the above two solutions is represented by

$$
D_{2}=M^{w}(0 \leq w<1),
$$

where $w=1$ is not included in its domain since $w=1$ corresponds to Case I $(\operatorname{see}(3))$. In SAS, users can set a value to $w$.

In addition to Cases I and II, we have Case III, which uses an orthogonal matrix $Q T^{*}$ with $T^{* \prime} T^{*}=I_{k}$ in place of $Q$ in Case II. The obliquely rotated loadings and the factor correlations become somewhat involved but can be obtained straightforwardly. The Case III solution was used by Hakstian and Abell (1974). However, the author has no knowledge about actual use of Case III other than that. Therefore, here only Case II is considered among orthoblique solutions.

\section{Covariance Structures for Orthoblique Solutions}

The parameters in (1) with (2) depend on the scales of the observed variables. However, in behavioral sciences standardized observed variables are usually used. For this case, (1) should be replaced by

$$
\begin{aligned}
\Sigma & =E\left(\Gamma+\operatorname{Diag}\left(I_{p}-\Gamma\right)\right) E \\
& =E \mathrm{P} E
\end{aligned}
$$


(see e.g., Jennrich, 1974), where $E(p \times p)$ is a diagonal matrix whose diagonal elements are the standard deviations of unstandardized observed variables and $P$ is a correlation matrix of the observed variables.

The parameters of interest are $B, \Phi$ and $\Psi$ which describe the model of unstandardized variables (see (1)), and $B, \Phi$ and $E$ for the model of standardized variables (see $(7)$ ). The asymptotic standard errors of the parameter estimates are given from the asymptotic variance-covariance matrix of the parameter estimates. However, it is not easy to obtain them directly by the usual method using the information matrix with restrictions among the parameters since the obliquely rotated parameters by the orthoblique method are given by the stepwise method mentioned previously.

Thus, we employ the following method for deriving the asymptotic standard errors of the parameter estimates:

Step 1. Instead of $B$ and $\Phi$ for the Case II solution, we use a reparameterized matrix $U\left(Q M^{w} T\right.$ ) (an orthogonally rotated matrix by $T$ ) with associated matrices $Q, M, T, \Psi$, (or $E$ ). It is not difficult to obtain the restrictions for $U$ since $U$ is an orthogonally rotated loading matrix with an analytical criterion such as the varimax criterion.

Step 2. The asymptotic variance-covariance matrix for $\hat{U}$ and other associated parameter estimates is given by the inverse of the augmented information matrix for the reparameterized matrices $Q, M, T, U, \Psi$, (or $E$ ).

Step 3. Since the matrices $B$ and $\Phi$ are functions of the reparameterized matrices, the asymptotic variance-covariance matrix of $\hat{B}$ and $\hat{\Phi}$ is obtained from the asymptotic variance-covariance matrix of the estimates of the reparameterized matrices by using the delta method.

Step 1. The matrix $\Gamma$ in (1) and (7) can be transformed into the following asymmetric matrix which includes both the unrotated and orthogonally rotated results for Case II:

$$
\Gamma=Q M^{2-w} T U^{\prime} \text { with } U=Q M^{w} T
$$

where $U$ is an orthogonally rotated loading matrix without rescaling factor $D_{1}$. Rewriting $D_{1}$ as simply $D$,

$$
\begin{aligned}
& D=\left[\operatorname{Diag}\left(T^{\prime} M^{2-2 w} T\right)\right]^{1 / 2}, \\
& B=U D \text { and } \\
& \Phi=D^{-1} T^{\prime} M I^{2-2 w} T D^{-1}
\end{aligned}
$$

follow. The obliquely rotated loading matrix $B$ is a rescaled version of $U$. Note that an optimizing function $h(U)$ of the orthogonal rotation (e.g., the varimax 
rotation) given by $T$ is with respect to $U$ rather than $B$. This is the reason for inclusion of $U$ in (8).

The parameter estimates whose standard errors are to be estimated are the non-fixed and non-duplicated elements of $\hat{B}, \hat{\Phi}$ and $\hat{\Psi}$ (or $\hat{E}$ ). Since $B$ and $\Phi$ are functions of $T, M$ and $U$ (see (9)), the asymptotic variances-covariances for the estimators $\hat{B}$ and $\hat{\Phi}$ are obtained if those for $\hat{T}, \hat{M}$ and $\hat{U}$ are given. The asymptotic covariance matrix for $\hat{T}, \hat{M}$ and $\hat{U}$ with associated matrices $\hat{Q}, \hat{\Phi}$ (or $\hat{E}$ ) is derived from the covariance structures (1) or (7) with $\Gamma$ replaced by (8).

\section{Asymptotic Standard Errors for Rotated Parameter Estimates}

Step 2. Let the following equation be a general restriction for the reparameterized parameters:

$$
\boldsymbol{g}(Q ; M ; T ; U ; \boldsymbol{\Psi}(\text { or } E))=\mathbf{0},
$$

and $I_{A}$ be an augmented information matrix for the parameters with the assumption of the multivariate normal distribution. Then, the submatrix $I^{*}(\cdot)$ in the inverse of $I_{A}$,

$$
\begin{aligned}
I_{A}^{-1} & =\left[\begin{array}{cc}
I(Q ; M ; T ; U ; \Psi(\text { or } E)), & \frac{\partial \boldsymbol{g}^{\prime}}{\partial \boldsymbol{\theta}} \\
\frac{\partial \boldsymbol{g}}{\partial \boldsymbol{\theta}^{\prime}}, & O
\end{array}\right]^{-1} \\
& =\left[\begin{array}{cc}
I^{*}(Q ; M ; T ; U ; \Psi(\text { or } E)), & \# \\
\#, & \#
\end{array}\right]
\end{aligned}
$$

is the asymptotic covariance matrix of the parameter estimates (see e.g., Silvey, 1975), where $I(\cdot ; \ldots ; \cdot)$ is the information matrix for the parameters in parentheses and is shown in Appendix 1; $\theta$ is the column vector consisting of the parameters in the model; and \#'s indicate submatrices which will not be used here.

The constraints (10) on the parameters and the partial derivatives in (11) are obtained as follows. From $U=Q M^{w} T$ the first restriction is written as

$$
\boldsymbol{g}_{1}=\operatorname{vec} G_{1}=\operatorname{vec}\left(U-Q M^{w} T\right)=\mathbf{0},
$$

where $\operatorname{vec}(\cdot)$ denotes a column vector consisting of the columns of the parenthesized matrix in consecutive order. The restriction comes from the redundancy of including $U$ for the description of the covariance structure models (1) and (7). Let $g_{1 i j}=\left(G_{1}\right)_{i j}$, where $(\cdot)_{i j}$ indicates the $(i, j)$ th element of the matrix in parentheses. Then,

$$
\begin{aligned}
& \frac{\partial g_{1 i j}}{\partial u_{s t}}=\delta_{i s} \delta_{j t},(i, s=1, \ldots, p ; j, t=1, \ldots, k), \\
& \frac{\partial g_{1 i j}}{\partial q_{s t}}=-\delta_{i s}\left(M^{w} T\right)_{t j},(i, s=1, \ldots, p ; j, t=1, \ldots, k),
\end{aligned}
$$




$$
\begin{aligned}
& \frac{\partial g_{1 i j}}{\partial m_{s s}}=-q_{i s} w m_{s s}^{w-1} t_{s j},(i=1, \ldots, p ; j, s=1, \ldots, k), \\
& \frac{\partial g_{1 i j}}{\partial t_{s t}}=-\delta_{j t}\left(Q M^{w}\right)_{i s},(i=1, \ldots, p ; j, s, t=1, \ldots, k),
\end{aligned}
$$

where $\delta_{i s}$ is the Kronecker delta $\left(\delta_{i s}=1, i=s ; \delta_{i s}=0, i \neq s\right)$.

The second restriction, which is from $Q^{\prime} Q=I_{k}$, is described as

$$
g_{2}=\mathrm{v} G_{2}=\mathrm{v}\left(Q^{\prime} Q-I_{k}\right)=\mathbf{0},
$$

where $\mathrm{v}(\cdot)$ denotes a column vector consisting of the elements on or below the diagonal elements of the parenthesized matrix. Let $g_{2 i j}=\left(G_{2}\right)_{i j}$, then

$$
\begin{aligned}
& \frac{\partial g_{2 i j}}{\partial q_{s t}}=\left(I_{t s} Q+Q^{\prime} I_{s t}\right)_{i j}=\delta_{i t} q_{s j}+\delta_{j t} q_{s i} \\
& (i \geq j ; s=1, \ldots, p ; t=1, \ldots, k) .
\end{aligned}
$$

The third restriction from $T^{\prime} T=I_{k}$ is

$$
\boldsymbol{g}_{3}=\mathrm{v} G_{3}=\mathrm{v}\left(T^{\prime} T-I_{k}\right)=\mathbf{0}
$$

Let $g_{3 i j}=\left(G_{3}\right)_{i j}$, then

$$
\frac{\partial g_{3 i j}}{\partial t_{s t}}=\delta_{i t} t_{s j}+\delta_{j t} t_{s i},(i \geq j ; s, t=1, \ldots, k) .
$$

The fourth restriction is for $U$ which optimizes an analytical rotation criterion $h(U)$. From Archer and Jennrich (1973), the restriction is described as

$$
\boldsymbol{g}_{4}=\mathrm{vb}\left(U^{\prime} \frac{\partial h(U)}{\partial U}-\frac{\partial h(U)}{\partial U^{\prime}} U\right)=\mathbf{0}
$$

where $\mathrm{vb}(\cdot)$ denotes a column vector consisting of the off-diagonal elements below the main diagonal of the parenthesized matrix. The actual expressions for (18) are given by Archer and Jennrich (1973) and Ogasawara (1996) for the raw-and normalized-orthomax methods, respectively. From the above results, the overall restriction

$$
\boldsymbol{g}=\left(\boldsymbol{g}_{1}^{\prime}, \boldsymbol{g}_{2}^{\prime}, \boldsymbol{g}_{3}^{\prime}, \boldsymbol{g}_{4}^{\prime}\right)^{\prime}=\mathbf{0}
$$

follows.

The numbers of non-fixed and non-duplicated parameters in (1) or (7) with (8) are $p k, k, k^{2}, p k$ and $p$ for $Q, M, T, U$ and $\Psi$ (or $E$ ), respectively. Thus, the total number of the parameters is $2 p k+p+k^{2}+k$. On the other hand, the number of restrictions are $p k,(1 / 2)\left(k^{2}+k\right),(1 / 2)\left(k^{2}+k\right)$ and $(1 / 2)\left(k^{2}-k\right)$ corresponding to the four subvectors in (19), respectively. The total number of the restrictions becomes $p k+(1 / 2)\left(3 k^{2}+k\right)$. Therefore, the size of the augmented 
information matrix, which is the sum of the above two total values, is $3 p k+p+$ $(1 / 2)\left(5 k^{2}+3 k\right)$, while the number of independent parameters, which is the number of the parameters minus that of the restrictions, is $p k+p-(1 / 2)\left(k^{2}-k\right)$ which corresponds to the number of independent parameters in an ordinary exploratoryfactor-analysis model.

Step 3. The asymptotic variances for the estimates of the obliquely rotated parameters $B$ and $\Phi$ are derived by using the delta method. That is, the asymptotic covariances for $\hat{\beta}_{i j}$ and $\hat{\beta}_{s t}$ (or for $\hat{\phi}_{i j}$ and $\left.\hat{\phi}_{s t}\right)$ denoted by acov $\left(\hat{\beta}_{i j}, \hat{\beta}_{s t}\right)$ (or acov $\left.\left(\phi_{i j}, \phi_{s t}\right)\right)$ are

$$
\begin{aligned}
& \operatorname{acov}\left(\hat{\beta}_{i j}, \hat{\beta}_{s t}\right)=\frac{\partial \beta_{i j}}{\partial \mathrm{p}(U, M, T)^{\prime}} \operatorname{acov}(\hat{U} ; \hat{M} ; \hat{T}) \frac{\partial \beta_{s t}}{\partial \mathrm{p}(U, M, T)}, \\
& (i, s=1, \ldots, p ; j, t=1, \ldots, k) \\
& \operatorname{acov}\left(\hat{\phi}_{i j}, \hat{\phi}_{s t}\right)=\frac{\partial \phi_{i j}}{\partial \mathrm{p}(M, T)^{\prime}} \operatorname{acov}(\hat{M} ; \hat{T}) \frac{\partial \phi_{s t}}{\partial \mathrm{p}(M, T)}, \\
& (i>j ; s>t)
\end{aligned}
$$

where $\operatorname{acov}(\hat{U} ; \hat{M} ; \hat{T})$ and $\operatorname{acov}(\hat{M} ; \hat{T})$ are the asymptotic covariance matrix among the estimates of the parameters of the parenthesized matrices, which have been obtained in Step 2 , and $\mathrm{p}(\cdot, \ldots, \cdot)$ denotes a column vector consisting of the non-fixed parameters in the parenthesized matrices. Replacing the true values of the parameters in (20) by their estimates, we have the estimates of the asymptotic covariance matrix for the estimates of the parameters. The partial derivatives in (20) are given in Appendix 2.

\section{Numerical Examples}

Numerical examples are based on two real correlation matrices. The first one is the correlation matrix of Lawley and Maxwell's (1971, p.66) six school subjects $(N=220)$. The second one is the correlation matrix of Harman's $(1976$, p.22) eight physical variables $(N=305)$. The number of common factors is assumed to be two for each example. When a model for unstandardized variables is fitted, the correlation matrices are supposed to be covariance matrices. Tables 1-4 give rotated results which include independent cluster solutions $(w=0)$ and proportional solutions $(w=.5)$, where the initial factor loading matrix was estimated by the maximum likelihood method, then $\hat{Q} \hat{M}^{2} \hat{Q}^{\prime}$ was obtained as the spectral decomposition of $\hat{\Gamma}$. The normalized varimax method was used for the orthogonal rotation applied to $\hat{Q} \hat{M}^{w}$. Note that the solutions in the tables are common solutions for unstandardized and standardized variables since the sample variances were assumed to be unities. 
Table 1: Results of independent cluster solution $(w=0)$ for six school subjects

\begin{tabular}{|c|c|c|c|c|c|c|}
\hline \multirow{3}{*}{\multicolumn{2}{|c|}{$\begin{array}{l}\text { Vari- } \\
\text { able } \\
\text { No. }\end{array}$}} & \multirow{3}{*}{ Estimates } & \multicolumn{2}{|c|}{ Unstandardized variables } & \multicolumn{2}{|c|}{ Standardized variables } \\
\hline & & & $N=220$ & $N=440$ & $N=220$ & $N=440$ \\
\hline & & & $(. S E S D)$ & $(S E S D)$ & $(S E S D)$ & $(S E S D)$ \\
\hline \multirow{6}{*}{ I } & 1 & .0083 & $(.0730 .0777)$ & $(.0515 \quad .0529)$ & $(.0728 .0775)$ & $(.0514 .0528)$ \\
\hline & 2 & & $.0754 .0869)$ & $(.0532 .05$ & $0749.0856)$ & $0529.0565\}$ \\
\hline & 3 & -.13 & $(.0793 .0827)$ & $(.0560 .0583)$ & $(.0790 .0823)$ & $(.0558 .0581)$ \\
\hline & 4 & .8179 & $(.0947 .0992)$ & $(.0669 .0663)$ & $(.0795 .0843)$ & $(.0562 \quad .0561)$ \\
\hline & 5 & .7468 & $(.0893 .0890)$ & $\left(\begin{array}{ll}.0631 & .0641\end{array}\right)$ & $(.0747 .0783)$ & $(.0528 \quad .0554)$ \\
\hline & & & $(.0838 .0852)$ & $(.0592 .0607)$ & $(.0728 .0751)$ & $(.0514 .0534)$ \\
\hline \multirow{6}{*}{ II } & & & $(.1088 .1168)$ & $(.0768 .0800)$ & $(.0978 .1054)$ & $(.0691 .0731)$ \\
\hline & 2 & & $(.0955 \quad .1032)$ & $(.0675 .073$ & $(.0873 .0990)$ & $(.0617 \quad .0664)$ \\
\hline & 3 & & $(.1100 .1163)$ & $(.0777 .079$ & $(.1000 .1092)$ & $(.0706 .0738)$ \\
\hline & & & $(.0722 \quad .0750)$ & $(.0510 .05$ & $(.0721 \quad .0748)$ & $(.0509 .0511)$ \\
\hline & 5 & & $(.0683 .072$ & $(.0483 .05$ & $(.0683 .0722)$ & $(.0482 \quad .0519)$ \\
\hline & 6 & .0593 & $(.0709 .0749)$ & $(.0501 \quad .0518)$ & $(.0708 .0746)$ & $(.0500 \quad .0518)$ \\
\hline \multicolumn{2}{|c|}{$\phi_{21}$} & .5680 & $(.0737 .0705)$ & $(.0521 \quad .0517)$ & $(.0729 .0693)$ & $(.0515 .0510)$ \\
\hline
\end{tabular}

Note.I=Factor I,II=Factor II, $\overline{S E}=$ standard error, $S D=$ standard deviation

Table 2: Results of proportional solution $(w=.5)$ for six school subjects

\begin{tabular}{|c|c|c|c|c|c|c|}
\hline \multirow{3}{*}{\multicolumn{2}{|c|}{$\begin{array}{l}\text { Vari- } \\
\text { able } \\
\text { No. }\end{array}$}} & \multirow{3}{*}{ Estimates } & \multicolumn{2}{|c|}{ Unstandardized variables } & \multicolumn{2}{|c|}{ Standardized variables } \\
\hline & & & $N=220$ & $N=440$ & $N=220$ & $N=440$ \\
\hline & & & $(S E S D)$ & $(S E S D)$ & $(S E S D)$ & $(S E S D)$ \\
\hline \multirow{6}{*}{ I } & 1 & .1301 & $(.0601 .0658)$ & $(.0424 .0440)$ & $(.0594 .0650)$ & $(.0419 .0434)$ \\
\hline & 2 & .2405 & $(.0709 .0773)$ & $(.0501 .0517)$ & $(.0691 \quad .0747)$ & $(.0488 .0509)$ \\
\hline & 3 & 108 & $(.0599 .0616)$ & $(.0423 .0447)$ & $(.0599 .0615)$ & $(.0423 .0446)$ \\
\hline & 4 & & $(.0814 .0852)$ & $(.0575 .056$ & $(.0656 .0695)$ & $(.0463 .0464)$ \\
\hline & 5 & & $(.0782 .077$ & $(.0552 .056$ & $(.0631 .0665)$ & $(.0446 .0469)$ \\
\hline & 6 & & $(.0747 .07$ & $(.0528 \quad .054$ & $(.0631 \quad .0655)$ & $(.0446 .0465)$ \\
\hline \multirow{6}{*}{ II } & & & $(.0958 .104$ & 7.070 & $(.0853 .0933)$ & $(.0603 .0642)$ \\
\hline & 2 & & $(.0866 .0911)$ & $(.0612 .066$ & $(.0787 .0870)$ & $\left(\begin{array}{lll}.0556 & .0594)\end{array}\right.$ \\
\hline & 3 & .6001 & $(.0969 .1029)$ & $(.0685 .0699)$ & $(.0869 .0956)$ & $(.0614 .0644)$ \\
\hline & 4 & .0549 & $(.0570 .0586)$ & $(.0403 .0409)$ & $(.0569 \quad 0589)$ & $(.0402 .0408)$ \\
\hline & 5 & .10 & $(.0583 .0630)$ & $(.0412 .0448)$ & $(.0578 .0620)$ & $(.0408 .0445)$ \\
\hline & 6 & 1283 & $(.0649 .0686)$ & $(.0459 .0471)$ & $(.0643 .0678)$ & $(.0454 .0466)$ \\
\hline \multicolumn{2}{|c|}{$\overline{\phi_{21}}$} & .3097 & $(.0491 \quad .0464)$ & $(.0347 .0341)$ & $(.0486 .0457)$ & $(.0343 \quad .0337)$ \\
\hline
\end{tabular}

Note.I $=$ Factor I,II =Factor II, $S E=$ standard error, $S D=$ standard deviation 
Table 3: Results of independent cluster solution $(w=0)$ for eight physical variables $(N=305)$

\begin{tabular}{|c|c|c|c|c|}
\hline \multicolumn{3}{|c|}{ Variable } & Unstandardized variables & \multirow{2}{*}{$\begin{array}{c}\text { Standardized variables } \\
(S E S D)\end{array}$} \\
\hline & & Estimates & $(S E S D)$ & \\
\hline \multirow{8}{*}{ I } & 1 & .8688 & $(.0448 .0439)$ & $(.0185 .0184)$ \\
\hline & 2 & .9693 & $(.0455 .0441)$ & $(.0143 .0146)$ \\
\hline & 3 & .9345 & $(.0468 .0464)$ & $(.0168 .0171)$ \\
\hline & 4 & .8723 & $(.0461 .0464)$ & $(.0193 .0205)$ \\
\hline & 5 & -.0180 & $(.0287 .0286)$ & $(.0287 .0286)$ \\
\hline & 6 & -.0157 & $(.0351 .0353)$ & $(.0351 .0352)$ \\
\hline & 7 & -.0758 & $(.0373 .0381)$ & $(.0372 \quad .0380)$ \\
\hline & 8 & $.1 \geqslant 16$ & $(.0431 .0438)$ & $(.0428 .0436)$ \\
\hline \multirow{8}{*}{ II } & 1 & .0819 & $(.0251 .0259)$ & $(.0250 .0256)$ \\
\hline & 2 & -.0528 & $(.0219 .0219)$ & $(.0219 .0219)$ \\
\hline & 3 & -.0460 & $(.0246 .0251)$ & $(.0246 .0251)$ \\
\hline & 4 & .0446 & $(.0261 \quad .0264)$ & $(.0260 .0266)$ \\
\hline & 5 & .9630 & $(.0487 .0499)$ & $(.0240 .0247)$ \\
\hline & 6 & .8051 & $(.0530 .0537)$ & $(.0311 .0319)$ \\
\hline & 7 & .7977 & $(.0547 .0561)$ & $(.0333 .0343)$ \\
\hline & 8 & .6135 & $(.0549 .0540)$ & $(.0415 .0412)$ \\
\hline \multicolumn{2}{|c|}{$\phi_{21}$} & .4837 & $(.0487 .0479)$ & $(.0483 .0476)$ \\
\hline
\end{tabular}

Note.I=Factor $\mathbf{I}, \mathrm{II}=$ Factor II, $S E=$ standard error, $S D=$ standard deviation

Table 4: Results of proportional solution $(w=.5)$ for eight physical variables $(N=305)$

\begin{tabular}{|c|c|c|c|c|}
\hline \multicolumn{3}{|c|}{ Variable } & Unstandardized variables & \multirow{2}{*}{$\begin{array}{c}\text { Standardized variables } \\
(S E S D)\end{array}$} \\
\hline & & Estimates & $(S E S D)$ & \\
\hline \multirow{8}{*}{ I } & 1 & .8483 & $(.0422 .0415)$ & $(.0172 .0168)$ \\
\hline & 2 & .9270 & $(.0421 .0406)$ & $(.0120 .0120)$ \\
\hline & 3 & .8943 & $(.0434 .0432)$ & $(.0144 .0148)$ \\
\hline & 4 & .8467 & $(.0433 .0434)$ & $(.0176 .0187)$ \\
\hline & 5 & .1133 & $(.0268 .0271)$ & $(.0257 .0261)$ \\
\hline & 6 & .0942 & $(.0342 .0339)$ & $(.0337 .0334)$ \\
\hline & 7 & .0352 & $(.0353 .0359)$ & $(.0353 .0359)$ \\
\hline & 8 & .2004 & $(.0431 .0434)$ & $(.0415 .0421)$ \\
\hline \multirow{8}{*}{ II } & 1 & .1794 & $(.0270 .0281)$ & $(.0254 .0263)$ \\
\hline & 2 & .0624 & $(.0230 .0224)$ & $(.0227 .0222)$ \\
\hline & 3 & .0647 & $(.0254 .0262)$ & $(.0251 \quad .0256)$ \\
\hline & 4 & .1442 & $(.0276 .0271)$ & $(.0265 .0264)$ \\
\hline & 5 & .9189 & $(.0440 .0453)$ & $(.0207 .0215)$ \\
\hline & 6 & .7682 & $(.0490 .0497)$ & $(.0277 .0284)$ \\
\hline & 7 & .7541 & $(.0506 .0517)$ & $(.0296 .0304)$ \\
\hline & 8 & .6009 & $(.0513 .0503)$ & $(.0378 .0375)$ \\
\hline \multicolumn{2}{|c|}{$\phi_{21}$} & .2578 & $(.0296 .0292)$ & $(.0294 .0290)$ \\
\hline
\end{tabular}


In Tables 1-4 results by simulation are included and obtained in the following way. First, the correlation matrices for the observed variables constructed by the estimated parameters, were regarded as population covariance matrices. From the population matrices, random observations of the same sample size as that of each data set or an assumed sample size (explained later) were generated with the assumption of the multivariate normal distribution. From the sample covariance (or correlation) matrix obtained from the generated observations. the values of the parameters in each model were estimated. This was repeated 1,000 times and 1.000 estimates were obtained for each parameter. The $S D$ in Tables 1-4 are their standard deviations. The Heywood cases are not included in the results by simulation. The maximum and minimum numbers of Heywood cases until 1,000 regular estimates were obtained in the sets of simulated data are twelve and zero, respectively. For unrotated and rotated solutions, there remain the indeterminacies of signs and permutations in the columns of loading matrices. They are removed by the method of Clarkson (1979), which searches for the pattern most similar to the population pattern.

Tables 1 and 2 show the results for the six school subjects. The factor correlation for the independent cluster solution $(w=0)$ is larger than that for the proportional solution $(w=.5)$, which is a general tendency (Hakstian, 1971; Hakstian \& Abell, 1974). For this case, a simpler pattern is obtained by the independent cluster solution than by the proportional solution. Overall, the theoretical standard errors for the rotated loadings in the case of the actual sample size seem to be similar to those by simulation considering the rather small sample size $(N=220)$ for this example. However, some of the theoretical standard errors with the actual sample size especially those for small loadings are somewhat underestimated. To see the effect of the sample size, the real sample size $(N=220)$ was doubled and the theoretical and simulated values were similarly computed. Tables 1 and 2 include the additional results for $N=440$. Note that the theoretical standard errors when $N=440$ are $\sqrt{219 / 439}(\cong .7063) \times$ [the theoretical standard errors when $N=220$ ]. The tables show that the differences between the theoretical and simulated values have been reduced by doubling the sample size. The standard errors for the estimates of the loadings for standardized variables are expected to be smaller than those for unstandardized variables since the loadings for standardized variables are restricted such that the diagonal elements of the correlation matrix for standardized variables are always one. The results show these tendencies. Especially for large loadings, the differences between their standard errors for unstandardized variables and those for standardized ones are notable.

Tables 3 and 4 show the results for the eight physical variables. The independent cluster solution gives more correlated factors and a simpler pattern than the proportional solution, a tendency similar to that for the six school subjects. The theoretical standard errors are very close to those by simulation. For the difference of the results for unstandardized and standardized variables, note that some of the theoretical and simulated standard errors for the large loadings for 
standardized variables are about one third of those for unstandardized variables.

\section{Discussion}

By employing the expression of (8), the asymptotic covariance matrix of the estimates of the reparameterized parameters was given. However, the size of the augmented information matrix becomes rather large by including the new parameter matrix $U$. The inverse of the augmented information matrix must be taken, though it is only once. The size $r$ of the matrix is equal to $3 p k+p+$ $(1 / 2)\left(5 k^{2}+3 k\right)$ as was shown previously and increases with the size of $p$ and/or $k$. The value $r$ as large as several hundreds may be quite feasible for present computers. (Several thousands may be possible depending on machines.) It is also possible to reduce the size by modifying (8) such as

$$
\Gamma=Q M^{2-w} T T^{\prime} M^{w} Q^{\prime}=Q M^{2} T T^{\prime} Q^{\prime} .
$$

Using (21), the reparameterized parameters are $Q, M, T$ and $\Psi$ (or $E$ ). Though the restriction $\boldsymbol{g}_{1}=\mathbf{0}$ is unnecessary for the expression of (21), the derivatives of the restriction $\boldsymbol{g}_{4}=\mathbf{0}$ with respect to the parameters become somewhat complicated since $g_{4}$ is now in terms of $Q, M$ and $T$ (see (18)). Let

$$
g_{4 i j}=\left(G_{4}\right)_{i j}=\left(U^{\prime} \frac{\partial h}{\partial U}-\frac{\partial h}{\partial U^{\prime}} U\right)_{i j},(k \geq i>j \geq 1),
$$

where $h=h(U)$, and a parameter in $\mathrm{p}(Q, M, T)$ be $\theta$. Then,

$$
\frac{\partial g_{4 i j}}{\partial \theta}=\left\{\frac{\partial U^{\prime}}{\partial \theta} \frac{\partial h}{\partial U}+U^{\prime} \partial\left(\frac{\partial h}{\partial U}\right) / \partial \theta-\left(\partial\left(\frac{\partial h}{\partial U^{\prime}}\right) / \partial \theta\right) U-\frac{\partial h}{\partial U^{\prime}} \frac{\partial U}{\partial \theta}\right\}_{i j}
$$

follows, where

$$
\left(\partial\left(\frac{\partial h}{\partial U}\right) / \partial \theta\right)_{s t}=\frac{\partial^{2} h}{\partial u_{s t}^{2}} \frac{\partial u_{s t}}{\partial \theta},(s=1, \ldots, p ; t=1, \ldots, k) .
$$

For this case, the number of the non-fixed parameters and that of the restrictions for the parameters are $p k+p+k^{2}+k$ and $(1 / 2)\left(3 k^{2}+k\right)$, respectively. Thus, $r=p k+p+(1 / 2)\left(5 k^{2}+3 k\right)$ which is less than the value for the previous model by $2 p k$.

As was described in the introduction, the method of deriving the asymptotic standard errors for the Case II solutions in factor analysis can be applied to the standard errors of rotated component loadings. This can be performed in the following way. For simplicity, we consider the case for unstandardized observed variables. The covariance structure for principal component analysis can be described as

$$
\Sigma=B \Phi B^{\prime}+\tilde{B} \tilde{B}^{\prime}=Q M^{2-w} T U^{\prime}+\tilde{B} \tilde{B}^{\prime}
$$


where $B \Phi B^{\prime}$ is a reproduced covariance matrix using the component loadings $B$ and the component correlations $\Phi$ of the first $k$ principal components followed by orthoblique rotation; and $\tilde{B} \tilde{B}^{\prime}$ is a residual matrix described by the component loading matrix $\tilde{B}$ of remaining $p-k$ orthogonal components. The form of the partial derivatives $\partial \boldsymbol{g} / \partial \boldsymbol{\theta}^{\prime}$ (see (11)) in the case of principal component analysis is similar to that for factor analysis with some additional restrictions such that $Q^{\prime} \tilde{B}=O(k \times(p-k))$ and $\tilde{B}^{\prime} \tilde{B}$ is diagonal. The information matrix should also be modified with $\Psi$ replaced by $\tilde{B} \tilde{B}^{\prime}$ in (11).

\section{REFERENCES}

Archer, C. O. \& Jennrich, R. I. (1973). Standard errors for rotated factor loadings. Psychometrika, 38, 581-592.

Browne, M. W. \& Du Toit, S. H. C. (1992). Automated fitting of nonstandard models. Multivariate Behavioral Research, 27, 269-300.

Clarkson, D. B. (1979). Estimating the standard errors of rotated factor loadings by jackknifing. Psychometrika, 44, 297-314.

Cudeck, R. \& O'Dell, L. L. (1994). Applications of standard error estimates in unrestricted factor analysis: Significance tests for loadings and correlations. Psychological Bulletin, 115, 475-487.

Efron, B. \& Tibshirani, R. J. (1993). An introduction to the bootstrap. New York: Chapman \& Hall.

Hakstian, A. R. (1971). A comparative evaluation of several prominent methods of oblique factor transformation. Psychometrika, 36, 175-193.

Hakstian, A. R. \& Abell, R. A. (1974). A further comparison of oblique factor transformation methods. Psychometrika, 39, 429-444.

Harman, H. H. (1976). Modern factor analysis (3rd ed.). Chicago: University of Chicago Press.

Harris, C. W. \& Kaiser, H. F. (1964). Oblique factor analytic solutions by orthogonal transformations. Psychometrika, 29, 347-362.

Jennrich, R. I. (1973). Standard errors for obliquely rotated factor loadings. Psychometrika, 38, 593-604.

Jennrich, R. I. (1974). Simplified formulae for standard errors in maximum likelihood factor analysis. British Journal of Mathematical and Statistical Psychology, 27, 122-131.

Kiers, H. A. L. (1997). Three-mode orthomax rotation. Psychometrika, 62, 579-598.

Kiers, H. A. L. \& Ten Berge, J. M. F. (1994). The Harris-Kaiser independent cluster rotation as a method for rotation to simple component weights. Psychometrika, 59, $81-90$.

Lawley, D. N. \& Maxwell, A. E. (1971). Factor analysis as a statistical method (2nd ed.). London: Butterworths.

Ogasawara, H. (1996). Standard errors for rotated factor loadings by normalized orthomax method. Japanese Joumal of Behaviormetrics, 23(2), 122-129. (in Japanese) 
Ogasawara, H. (1998). Standard errors for rotation matrices with an application to the promax solution. British Journal of Mathematical and Statistical Psychology, 51, $163-178$.

Ogasawara, H. (1999). Standard errors for procrustes solutions. Japanese Psychological Research, 41, 121-130.

SAS Institute Inc. (1990). SAS/STAT user's guide Vol.1 (version 6, 4th ed.). Cary, NC: Author.

Silvey, S. D. (1975). Statistical inference. New York: Chapman \& Hall.

Received October 1998. Revised August 1999

\section{Appendix 1. Information Matrix}

Let $L$ be the Wishart likelihood for the parameters in the covariance structure for unstandardized observed variables

$$
\Sigma=Q M^{2-w} T U^{\prime}+\Psi
$$

or the parameters in the covariance structure for standardized observed variables

$$
\Sigma=E\left(Q M^{2-w} T U^{\prime}+\operatorname{Diag}\left(I_{p}-Q M^{2-w} T U^{\prime}\right)\right) E
$$

Then,

$$
\ln L=-\frac{n}{2}\left[\ln |\Sigma|+\operatorname{tr}\left(S \Sigma^{-1}\right)\right]+\text { constant }
$$

where $n+1=N$ is the number of observations and $S$ is an unbiased sample covariance matrix. Let $\theta_{i}$ and $\theta_{j}$ be the $i$-th and $j$-th parameters in the model, respectively, then we have the $(i, j)$ th element of the information matrix:

$$
\begin{aligned}
I\left(\theta_{i}, \theta_{j}\right) & =\operatorname{Ex}\left(-\partial^{2} \ln L / \partial \theta_{i} \partial \theta_{j}\right) \\
& =\frac{n}{2} \operatorname{tr}\left(\Sigma^{-1} \frac{\partial \Sigma}{\partial \theta_{i}} \Sigma^{-1} \frac{\partial \Sigma}{\partial \theta_{j}}\right)
\end{aligned}
$$

where $\operatorname{Ex}(\cdot)$ denotes the expectation over the distribution of $S$. The matrices $\partial \Sigma / \partial \theta_{i}$ in (A4) for unstandardized variables are as follows:

$$
\begin{aligned}
& \frac{\partial \Sigma}{\partial q_{i j}}=I_{i j} M^{2-w} T U^{\prime}, \quad \frac{\partial \Sigma}{\partial m_{j j}}=(2-w) m_{j j}^{1-w} Q I_{j j} T U^{\prime} \\
& \frac{\partial \Sigma}{\partial t_{j l}}=Q M^{2-w} I_{j l} U^{\prime}, \quad \frac{\partial \Sigma}{\partial u_{i j}}=Q M^{2-w} T I_{j i} \\
& \frac{\partial \Sigma}{\partial \Psi_{i i}}=I_{i i}, \quad(i=1, \ldots, p: j, l=1, \ldots, k)
\end{aligned}
$$

where $I_{i j}$ denotes the matrix of an appropriate size whose $(i, j)$ th element is one and others are zero. The expressions for some of the pairs of the parameters in 
(A4) become simple by expanding the trace such as

$$
\frac{n}{2} \operatorname{tr}\left(\Sigma^{-1} \frac{\partial \Sigma}{\partial \Psi_{i i}} \Sigma^{-1} \frac{\partial \Sigma}{\partial \Psi_{j j}}\right)=\frac{n}{2}\left(\left(\Sigma^{-1}\right)_{i j}\right)^{2}
$$

where $(\cdot)_{i j}$ denotes the $(i, j)$ th element of the matrix in parentheses. However, (A4) with (A6) is sufficient for exact computation.

On the other hand, the matrices $\partial \Sigma / \partial \theta_{i}$ for standardized observed variables are as follows (see (A2)):

$$
\begin{aligned}
& \frac{\partial \Sigma}{\partial q_{i j}}=E\left(I_{i j} M^{2-w} T U^{\prime}-\left(M^{2-w} T U^{\prime}\right)_{j i} I_{i i}\right) E \\
& \frac{\partial \Sigma}{\partial m_{j j}}=(2-w) m_{j j}^{1-w} E\left(Q I_{j j} T U^{\prime}-\operatorname{Diag}\left(Q I_{j j} T U^{\prime}\right)\right) E \\
& \frac{\partial \Sigma}{\partial t_{j l}}=E\left(Q M^{2-w} I_{j l} U^{\prime}-\operatorname{Diag}\left(Q M^{2-w} I_{j l} U^{\prime}\right)\right) E \\
& \frac{\partial \Sigma}{\partial u_{i j}}=E\left(Q M^{2-w} T I_{j i}-\left(Q M^{2-w} T\right)_{i j} I_{i i}\right) E \\
& \frac{\partial \Sigma}{\partial e_{i i}}=I_{i i} \mathrm{P} E+E \mathrm{P} I_{i i}, \quad(i=1, \ldots, p ; j, l=1, \ldots, k) .
\end{aligned}
$$

\section{Appendix 2. Partial Derivatives of the Rotated Parameters}

The loadings and correlations of the obliquely rotated factors by the Case II orthoblique method are

$$
\begin{aligned}
B & =U D=U\left[\operatorname{Diag}\left(T^{\prime} M^{2-2 w} T\right)\right]^{1 / 2} \text { and } \\
\Phi & =D^{-1} T^{\prime} M^{2-2 w} T D^{-1} \\
& =\left[\operatorname{Diag}\left(T^{\prime} M^{2-2 w} T\right)\right]^{-1 / 2} T^{\prime} M^{2-2 w} T\left[\operatorname{Diag}\left(T^{\prime} M^{2-2 w} T\right)\right]^{-1 / 2},
\end{aligned}
$$

respectively. The nonzero partial derivatives of $B$ and $\Phi$ with respect to the parameters employed in the covariance structures (1) or (7) with (8) are as follows:

$$
\begin{aligned}
\frac{\partial \beta_{i j}}{\partial u_{s t}} & =\delta_{i s} \delta_{j t}(D)_{j j}, \\
\frac{\partial \beta_{i j}}{\partial m_{s s}} & =\frac{1}{2}\left[U\left(\operatorname{Diag}\left(T^{\prime} M^{2-2 w} T\right)\right)^{-1 / 2}(2-2 w) m_{s s}^{1-2 w} \operatorname{Diag}\left(T^{\prime} I_{s s} T\right)\right]_{i j} \\
& =(1-u) m_{s s}^{1-2 w}\left[U D^{-1} \operatorname{Diag}\left(T^{\prime} I_{s s} T\right)\right]_{i j}, \\
\frac{\partial \beta_{i j}}{\partial t_{s t}} & =\frac{1}{2}\left[U\left(\operatorname{Diag}\left(T^{\prime} M^{2-2 w} T\right)\right)^{-1 / 2} \operatorname{Diag}\left(I_{t s} M^{2-2 w} T+T^{\prime} M^{2-2 w} I_{s t}\right)\right]_{i j} \\
& =\delta_{t j}\left(U D^{-1}\right)_{i t}\left(M^{2-2 w} T\right)_{s t}, \\
\frac{\partial \phi_{u v}}{\partial m_{s s}} & =\left[-\frac{1}{2} D^{-3}\left(2-2 u^{\prime}\right) m_{s s}^{1-2 w} \operatorname{Diag}\left(T^{\prime} I_{s s} T\right) T^{\prime} M^{2-2 w} T D^{-1}\right.
\end{aligned}
$$




$$
\begin{aligned}
& +D^{-1}(2-2 u) m_{s s}^{1-2 w} T^{\prime} I_{s s} T D^{-1} \\
& \left.-\frac{1}{2} D^{-1} T^{\prime} M^{2-2 w} T D^{-3}(2-2 u) m_{s s}^{1-2 w} \operatorname{Diag}\left(T^{\prime} I_{s s} T\right)\right]_{u v} \\
= & -(1-u) m_{s s}^{1-2 w}\left[D ^ { - 1 } \left\{D^{-2} \operatorname{Diag}\left(T^{\prime} I_{s s} T\right) T^{\prime} M^{2-2 w} T-2 T^{\prime} I_{s s} T\right.\right. \\
& \left.\left.\quad+T^{\prime} M^{2-2 w} T D^{-2} \operatorname{Diag}\left(T^{\prime} I_{s s} T\right)\right\} D^{-1}\right]_{u v} \\
\frac{\partial \phi_{u v}}{\partial t_{s t}}= & {\left[-\frac{1}{2} D^{-3} \operatorname{Diag}\left(I_{t s} M^{2-2 w} T+T^{\prime} M^{2-2 w} I_{s t}\right) T^{\prime} M^{2-2 w} T D^{-1}\right.} \\
& +D^{-1}\left(I_{t s} M^{2-2 w} T+T^{\prime} M^{2-2 w} I_{s t}\right) D^{-1} \\
& \left.-\frac{1}{2} D^{-1} T^{\prime} M^{2-2 w} T D^{-3} \operatorname{Diag}^{2-2}\left(I_{t s} M^{2-2 w} T+T^{\prime} M^{2-2 w} I_{s t}\right)\right]_{u v} \\
= & -\delta_{u t}\left(D^{-1}\right)_{t t}\left[\left(D^{-2}\right)_{t t}\left(M^{2-2 w} T\right)_{s t}\left(T^{\prime} M^{2-2 w} T\right)_{t v}-\left(M^{2-2 w} T\right)_{s v}\right]\left(D^{-1}\right)_{v v} \\
& -\delta_{v t}\left(D^{-1}\right)_{t t}\left[\left(D^{-2}\right)_{t t}\left(M^{2-2 w} T\right)_{s t}\left(T^{\prime} M^{2-2 w} T\right)_{t u}-\left(M^{2-2 w} T\right)_{s u}\right]\left(D^{-1}\right)_{u u}, \\
& (i=1, \ldots, p ; j, s, t=1, \ldots, k ; k \geq u>v \geq 1)
\end{aligned}
$$

